\title{
Dual-Band Stepped-Impedance Filters For Ultra-Wideband Applications
}

\author{
Marjan Mokhtaari ${ }^{\#}$, Jens Bornemann ${ }^{\#}$ and Smain Amari* \\ ${ }^{*}$ Department of Electrical and Computer Engineering, University of Victoria \\ PO Box 3055, Victoria, BC, V8W 3P6, Canada \\ * Department of Electrical and Computer Engineering, Royal Military College of Canada \\ 17000 Station Forces, Kingston, ON, K7K 7B4, Canada
}

\begin{abstract}
New ultra-wideband planar dual-band filter designs are presented. Two broad passbands with up to 48 percent fractional bandwidths are obtained by utilizing quarterwavelength coupled-line section to create additional reflection zeros within each passband. Parallel open-end high impedance segments in stepped-impedance resonators achieve passband separation as they provide attenuation poles between the passbands. Out-of-band rejection is improved by introducing source-load coupling, which produces attenuation poles in the lower and higher rejection bands. The basic design approach is discussed. Several different dual ultra-wideband filters are proposed. Two different professional EM software packages verify the individual responses.
\end{abstract}

\section{INTRODUCTION}

The definition of ultra-wideband (UWB) applications specifies that related systems be required to cover a minimum bandwidth of 25 percent [1]. Since the release of the $3.1-$ 10.6 GHz band, activities in planar filter design focused on the coverage of the entire 110-percent band, e.g. [2] -[4]. All of these designs employ the multiple resonance properties of stepped-impedance resonators (SIR's), which are also commonly used in dual-band filter applications, e.g. [5] - [7]. However, the dual-band filters presented so far are relatively narrowband and do not fall into the UWB category according to the above definition.

Therefore, this paper presents the design of new dual-band filters in which each passband covers a bandwidth of up to 48 percent. Compared to standard stepped-impedance dual-band filters, the extended bandwidths are achieved by using coupling elements formed by quarter-wavelength coupled-line sections as known from UWB filters. Different steppedimpedance resonator configurations and structural folding add to the compactness of the designs.

\section{DESIGN}

Fig. 1 depicts the evolution of stepped-impedance resonators from the basic configuration (Fig. 1a) to more sophisticated implementation (Fig. 1b-1e). The parallel openended high-impedance segments in Fig. $1 \mathrm{~b}$ and $1 \mathrm{c}$ are determined through partial impedance division - as are the more compact low-impedance sections in Fig. 1c and 1e. These SIR structures not only have the same capability of resonating and operating at fundamental and harmonic frequencies, but they also possess the flexibility to be arranged as in cascaded-SIR UWB filters [2], [4]. At the same time, however, they are capable of generating attenuation poles between the fundamental and first higher-order resonances. (d)

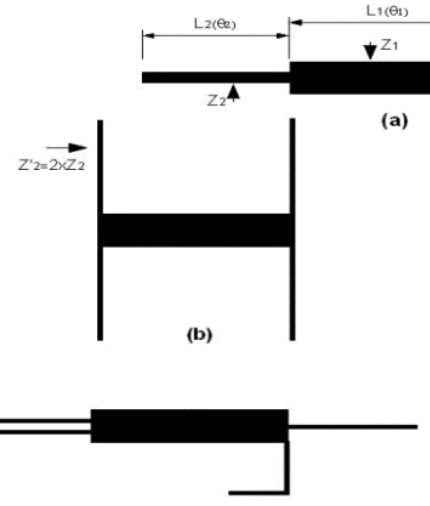

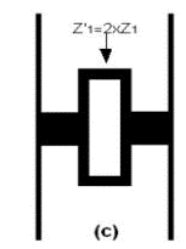

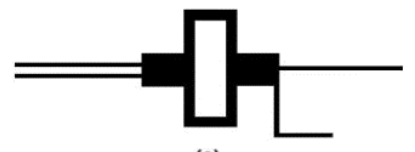

(e)
Fig. 1 Basic to advanced configurations of stepped-impedance resonators.

Fig. 2 shows the assorted coupled-line sections employed in the proposed dual UWB filter designs. The coupling from the input/output to the first/last resonator as shown in Fig. 2a is stronger than traditional ones and follows implementations as shown in, e.g., [8]. It not only reduces the requirement for high-accuracy fabrication but also provides strong input/output coupling which increases bandwidth. The quarter-wavelength coupled-line sections between resonators (Fig. 2b-2d) can generate an additional reflection zero within the passbands, thus increasing the order of the filter and hence the bandwidth.

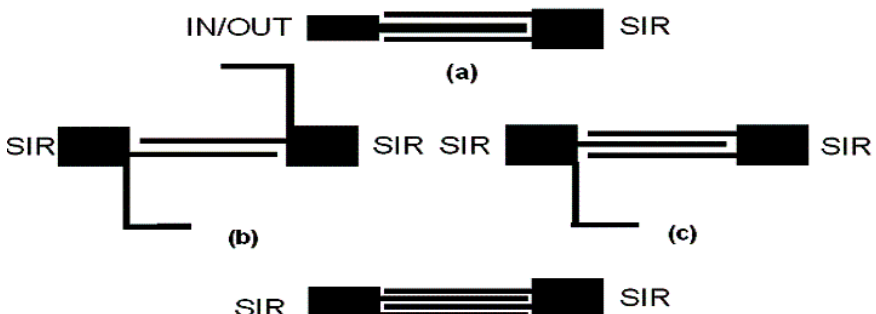

(d)

Fig. 2 Assorted coupled-line sections employed in dual-band UWB filter prototypes: Input/output coupling segment to the first/last SIR (a); coupledline section used in cascaded two-SIR (b), three-SIR (c) and four-SIR (d) filters.

A dual-band filter is normally expressed in terms of fractional bandwidth $(\Delta f)$ and the center frequencies in the first $\left(f_{1}\right)$ and second $\left(f_{2}\right)$ passbands [5]. The center frequency $\left(f_{0}\right)$ for the dual-band design is taken as the arithmetic mean of 
the two passband center frequencies. For the individual resonance frequencies of a basic SIR, the impedance ratio $R_{z}=Z_{2} / Z_{1}$ and the relationship between electrical section lengths $U=\theta_{2} /\left(\theta_{1}+\theta_{2}\right) \approx L_{2} /\left(L_{1}+L_{2}\right) \quad$ (Fig. 1a), the reader is referred to, e.g., [5]. The fundamental and higher-order mode resonances occur alternatively in the odd and even modes. The ratio of the harmonic frequencies to the fundamental-mode resonance can be specified in terms of $R_{z}$ and $U$.

Fig. 3 depicts the ratio of the harmonic frequencies to the fundamental one $\left(f_{n} / f_{1}\right)$ of a basic SIR in terms of length ratio $U$ for different impedance ratios $R_{z}$ [3]. In the design of dualband filters, the fundamental and first higher-order resonances are normally allocated as center frequencies in the first and second passband, respectively. In addition, the second harmonic should be moved away from the second passband in order to achieve wider upper-band rejection.

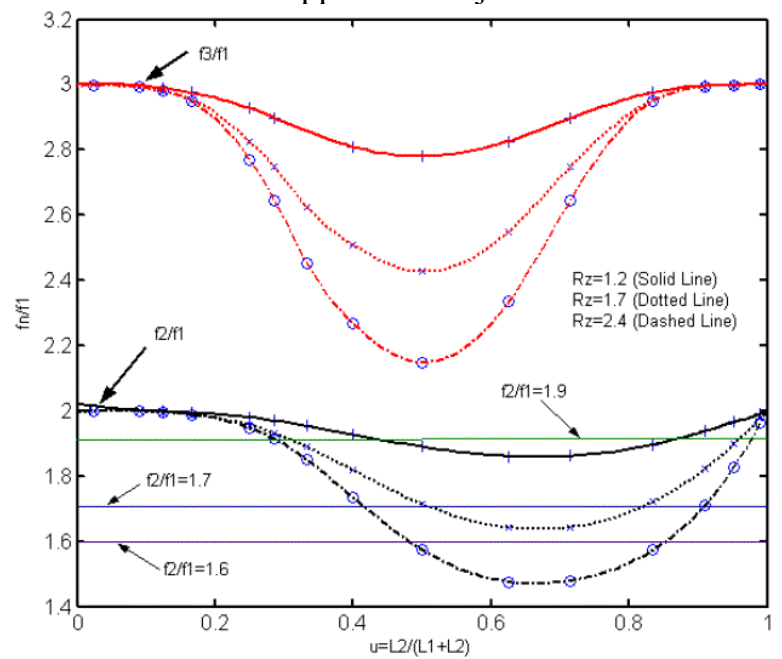

Fig. 3 Harmonic resonance frequency ratios of a basic SIR in terms of electrical length ratios and for different impedance ratios.

In order to achieve high isolation between passbands as well as an additional reflection zero in each passband, the electrical length of the open-ended high-impedance coupledline section $\left(\theta_{2}\right)$ must be $\pi / 2$ at center frequency $f_{0}$. Once the physical length $L_{2}$ is known, $L_{1}$ can be specified through the length ratio $U$. In this design approach, it is also assumed that the coupling coefficients between coupled-line sections are frequency independent. Furthermore, due to the additional reflection zero of the quarter-wavelength coupled-line sections, the number of required resonators is usually one less than that of a typical Chebychev passband filter design.

\section{RESULTS}

As a first example, let us design a dual-band filter with center frequencies at $f_{1}=2.2 \mathrm{GHz}, f_{2}=3.5 \mathrm{GHz}\left(f_{2} / f_{1} \approx 1.6\right)$ and $10 \mathrm{~dB}$ fractional bandwidth of $\Delta f=600 \mathrm{MHz}$ ( 28 percent) for the first and second pass-band. The center frequency of the entire filter is located at $f_{0}=2.85 \mathrm{GHz}$, which specifies the physical length of the high-impedance coupled-line segment $\left(L_{2}\right)$ to be a quarter-wavelength. Fig. 4 shows the response of the filter prototype as validated by two different EM field solvers (Ansoft Designer ${ }^{B}$ and IE3D $^{\circledR}$ ). Note that only two cascaded SIR's are used but that the filter is of order three in both passbands due to the parallel-line coupling sections. The filter prototype, as depicted in the inset of Fig. 4, is designed on RT6010 substrate with a height of 25 mils. Due to the frequency-dependent coupling coefficients (compared to constant ones assumed in the design), the bandwidth in the second passband is slightly higher than specified $(\sim 700 \mathrm{MHz})$. The third harmonic is located at $5.8 \mathrm{GHz}$ based on the values for impedance and length ratios. For this design, this is the largest possible distance from the second passband.

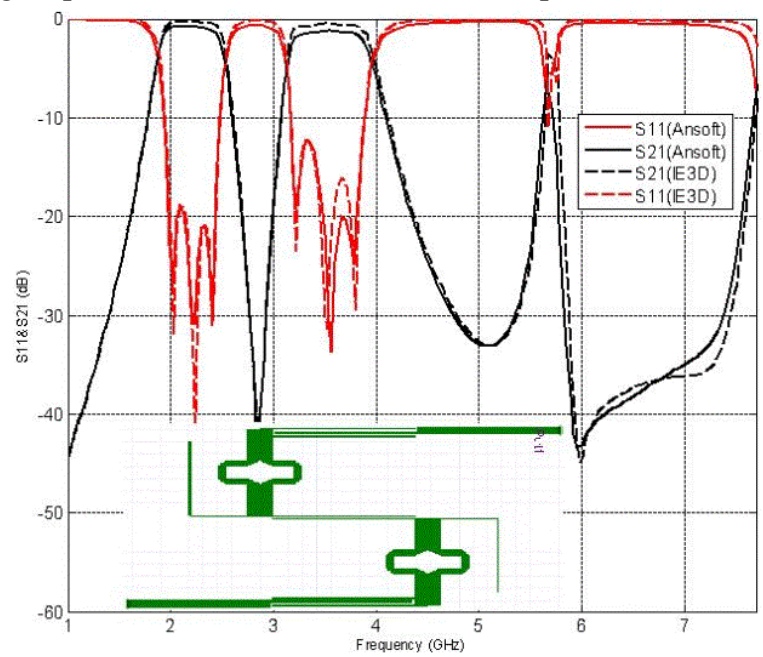

Fig. 4 Response of a three-pole dual-band filter $\left(f_{0}=2.85 \mathrm{GHz}\right)$ using two cascaded SIR's of Fig. 1e and coupled-line segments of Fig. $2 \mathrm{~b}$.

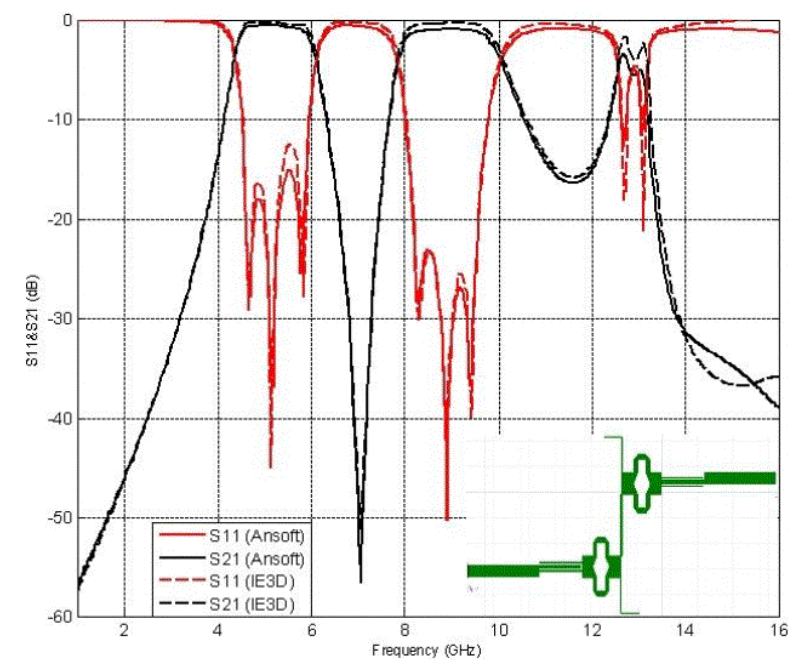

Fig. 5 Response of a three-pole dual-band filter $\left(f_{0}=7 \mathrm{GHz}\right)$ using two cascaded SIR's of Fig. 1e and coupled-line segments of Fig. 2b.

Fig. 5 shows a different three-pole, two-SIR dual-band filter topology with a center frequency of $f_{0}=7 \mathrm{GHz}$. The substrate is RT6006 with a height of 25 mils. The center frequencies for the first and second passbands are $f_{1}=5.25$ $\mathrm{GHz}$ and $f_{2}=8.85 \mathrm{GHz} \quad\left(f_{2} / f_{1} \approx 1.7\right)$, respectively. The bandwidths obtained in this design are approximately $1.5 \mathrm{GHz}$ for the first and $1.75 \mathrm{GHz}$ for the second passband. The return loss is better than $15 \mathrm{~dB}$, and the insertion loss is less than 1.5 $\mathrm{dB}$ in each passband.

In order to extend the fractional bandwidth in each passband, more resonators are required. A four-pole dual band 
filter prototype with three cascaded SIR's is shown in the inset of Fig. 6. It uses RT6006 substrate with $h=25$ mils. The design specifications are: $f_{1}=4.86 \mathrm{GHz}, f_{2}=9.15 \mathrm{GHz}\left(f_{2} / f_{1} \approx 1.9\right)$, and a $3 \mathrm{~dB}$ fractional bandwidth of $2 \mathrm{GHz}$ ( 42 percent). Fig. 6 shows the performance. In order to allocate the two resonances of the third (right-most) SIR within the two passbands, different impedance and length ratios are selected for this resonator. Otherwise, the use of the same SIR as the first and last resonators would create reflection zeros too close to the rejection band between the passbands.

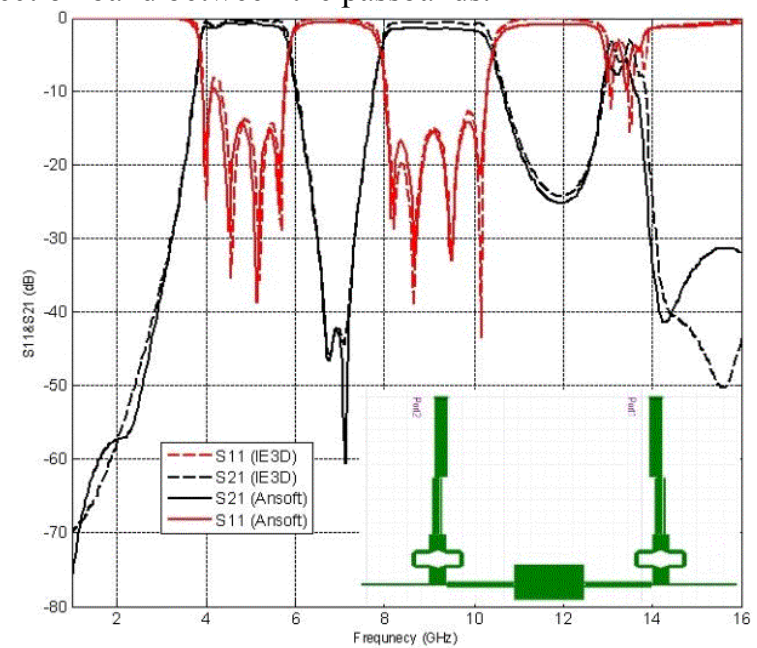

Fig. 6 Performance of a four-pole, three-SIR dual-band filter $\left(f_{0}=7 \mathrm{GHz}\right)$ using SIR's of Fig. 1b,1e and coupled-line segment of Fig. 2c.

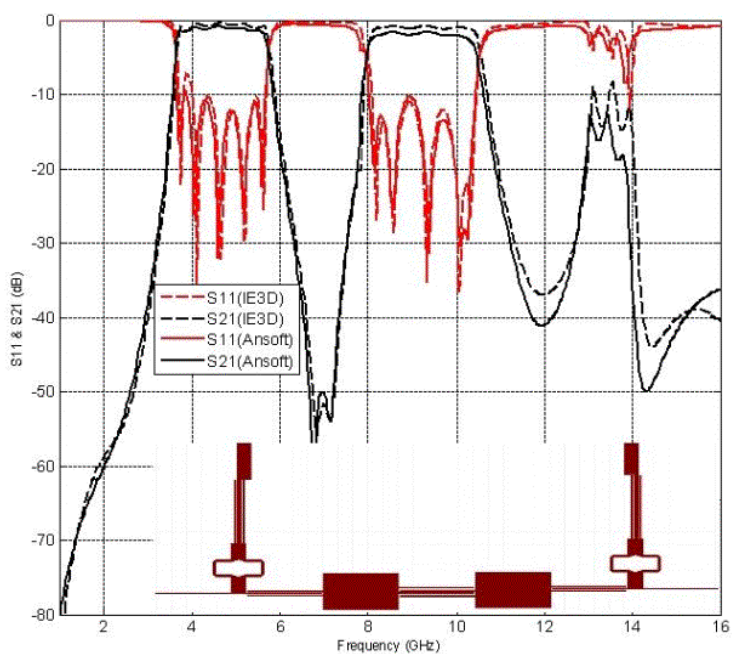

Fig. 7 Performance of a five-pole, four-SIR dual-band filter $\left(f_{0}=7 \mathrm{GHz},\right)$ using SIR's of Fig. 1b, 1e and coupled-line sections of Fig. 2c, 2d.

A five-pole dual-band filter approaching 48 percent fractional bandwidth including four cascaded SIR's can be set up as shown in the inset of Fig. 7. The center frequencies are selected at $f_{1}=4.7 \mathrm{GHz}$ and $f_{2}=9.2 \mathrm{GHz}\left(f_{2} / f_{1} 1 \approx 1.95\right)$. The simulated performances, as depicted in Fig. 6 , confirm $3 \mathrm{~dB}$ fractional bandwidths of $2.1 \mathrm{GHz}$ and $2.3 \mathrm{GHz}$. The insertion loss is less than $2 \mathrm{~dB}$, and the return loss is better than $10 \mathrm{~dB}$. The substrate used for this filter prototype is RT6006 with $h=25$ mils.
For the UWB dual-band filters in previous examples, the rejections beyond the second passbands are relatively low due to the third harmonic resonances. Therefore, attenuation poles are required in this frequency range in order to create a sharp skirt. This can be achieved through source-load coupling which is capable of generating such poles in both the lower and upper rejection bands. The locations of the attenuation poles can be changed by the source-load coupling coefficient.

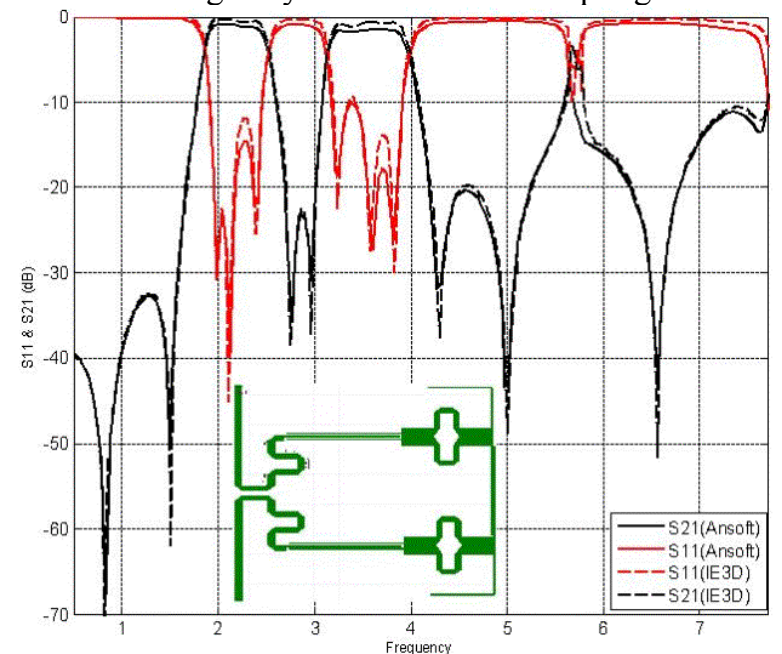

Fig. 8 Response of the source-load-coupled version of a dual-band filter similar to that of Fig. 4.

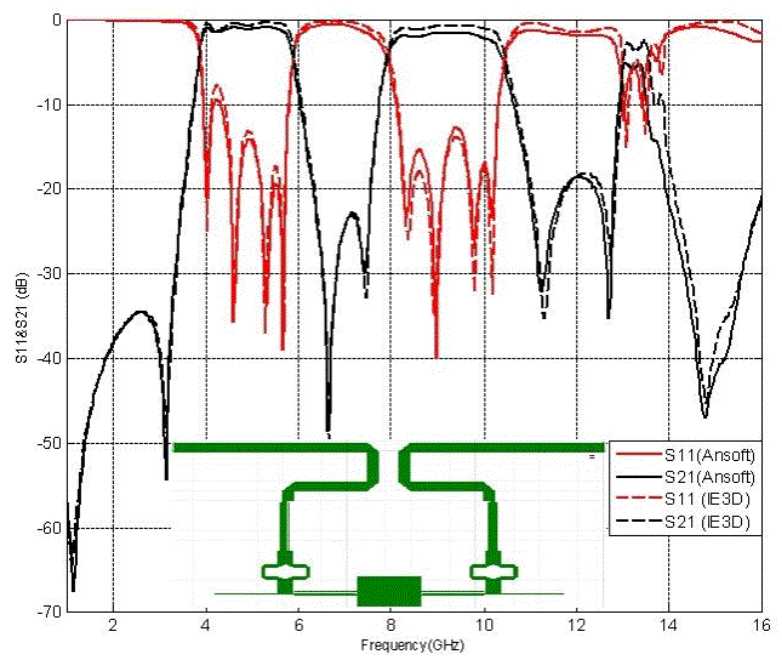

Fig. 9 Response of the source-load-coupled version of the dual-band filter presented in Fig. 6.

Fig. 8 shows the performance of a dual-band filter similar to that of Fig. 4 after introducing source-load coupling. The additional attenuation poles are produced at $800 \mathrm{MHz}, 1.5$ $\mathrm{GHz}, 4.3 \mathrm{GHz}$ and $5 \mathrm{GHz}$. Moreover, the double pole between the bands in Fig. 4 is now converted to two single ones at 2.8 $\mathrm{GHz}$ and $3 \mathrm{GHz}$. It appears that source-load coupling moves the attenuation poles between the bands slightly outward, which leads to higher selectivity for each passband.

Source-load coupling is also implemented for the dualband filter topology in Fig. 6. In comparison, Fig. 9 confirms additional attenuation poles at $1.2 \mathrm{GHz}, 3.2 \mathrm{GHz}, 11.4 \mathrm{GHz}$ and $12.6 \mathrm{GHz}$. Moreover, the two poles between the 
passbands moved slightly towards the passbands and are now located at $6.6 \mathrm{GHz}$ and $7.5 \mathrm{GHz}$.

Obviously, source-load coupling improves selectivity for each passband and creates sharp skirts in both the lower and upper stopband region.

\section{CONCLUSIONS}

New microstrip stepped-impedance resonator configurations are proposed for UWB dual-band filter applications. The designs employ a number of different SIR's and coupling sections. Parallel-coupled line sections cascade individual SIR's and, at the same time, increase the order of the filter by one in both passbands. Open-ended highimpedance segments are capable of generating attenuation poles between the two passbands. Attenuation poles towards lower and higher frequencies are generated by source-load coupling which also improves the attenuation between the passbands and, therefore, contributes to passband selectivity. The proposed dual-band filters provide ultra-wide fractional bandwidths between 28 and 48 percent in both passbands. The presented design examples are verified through results from two different professional electromagnetic field solvers.

\section{REFERENCES}

[1] B. Pattan, "A brief exposure to ultra-wideband signaling", Microwave J., Vol. 46, pp. 104-110, Dec. 2003

[2] L. Zhu, S. Sun and W. Menzel, "Ultra-wideband (UWB) bandpass filters using multiple-mode resonator", IEEE Microwave Wireless Comp. Lett., Vol. 15, pp. 796-798, Nov. 2005.

[3] Y.-C. Chiou, J.-T. Kuo, E. Chang, "Broadband quasi-Chebyshev bandpass filter with multimode stepped-impedance resonators (SIRs)", IEEE Trans. Microwave Theory Tech., Vol. 54, pp. 3352-3358, Aug. 2006.

[4] M.Mokhtaari, J.Bornemann, S.Amari, "Folded Compact ultrawideband stepped-impedance resonator filters", 2007 IEEE MTT-S Int. Microwave Symp. Dig., Honolulu, USA, June 2007.

[5] J.-T. Kuo, T.-H. Yeh and C.-C. Yeh, "Design of microstrip bandpass filters with a dual-passband response", IEEE Trans. Microwave Theory Tech., Vol. 53, pp. 1331-1337, Apr. 2005.

[6] J. Wang, Y.-X. Guo, B.Z. Wang and L.C. Ong, "Dual-band steppedimpedance band-pass filter", in Proc. 36th European Microwave Conf., pp. 902-904, Manchester, UK, Oct. 2006.

[7] M. Mokhtaari, J. Bornemann and S. Amari, "New reduced-size stepimpedance dual-band filters with enhanced bandwidth and stopband performance", in 2006 IEEE MTT-S Int. Microwave Symp. Dig., pp. 1181-1184, San Francisco, USA, June 2006.

[8] S. W. Wong, S. Sun, L. Zhu, and Z. N. Chen, "Ultra-wideband (UWB) bandpass filters with improved upper-stopband performance", Proc. 2006 Asia-Pacific Microwave Conf., Paper WE1B-2, Yokohama, Japan, Dec 2006. 